\title{
Pulmonary function and CT biomarkers as risk factors for cardiovascular events in male lung cancer screening participants: the NELSON study
}

\author{
Richard A. P. Takx • Rozemarijn Vliegenthart • Firdaus A. A. Mohamed Hoesein • \\ Ivana Išgum • Harry J. de Koning • Willem P. Th. M. Mali • Carlijn M. van der Aalst • \\ Pieter Zanen • Jan-Willem J. Lammers • Harry J. M. Groen • Eva M. van Rikxoort • \\ Michael Schmidt • Bram van Ginneken • Matthijs Oudkerk • Tim Leiner • Pim A. de Jong
}

Received: 21 March 2014 / Revised: 5 June 2014 / Accepted: 5 August 2014 / Published online: 3 September 2014

(C) European Society of Radiology 2014

\begin{abstract}
Objective The objective of this study was to investigate the association of spirometry and pulmonary CT biomarkers with cardiovascular events.

Methods In this lung cancer screening trial 3,080 male participants without a prior cardiovascular event were analysed. Fatal and non-fatal cardiovascular events were included. Spirometry included forced expiratory volume measured in units of one-second percent predicted ( $\mathrm{FEV}_{1} \%$ predicted) and $\mathrm{FEV}_{1}$ divided by forced vital capacity (FVC; $\mathrm{FEV}_{1} / \mathrm{FVC}$ ). CT examinations were quantified for coronary artery calcium volume, pulmonary emphysema (perc15) and bronchial wall thickness (pi10). Data were analysed via a Cox proportional hazard analysis, net reclassification improvement (NRI) and C-indices.
\end{abstract}

\footnotetext{
R. A. P. Takx $(\bowtie) \cdot$ F. A. A. M. Hoesein - W. P. T. M. Mali

T. Leiner $\cdot$ P. A. de Jong

Department of Radiology, University Medical Center Utrecht, Heidelberglaan 100, 3508 GA Utrecht, The Netherlands

P.O. Box 85500

e-mail: R.A.P.Takx@umcutrecht.nl

R. Vliegenthart $\cdot$ M. Oudkerk

Center for Medical Imaging - North East Netherlands, University of Groningen, University Medical Center Groningen, Groningen, The Netherlands

R. Vliegenthart

Department of Radiology, University of Groningen, University

Medical Center Groningen, Groningen, The Netherlands

I. Išgum

Images Sciences Institute, University Medical Center Utrecht,

Utrecht, The Netherlands
}

Results 184 participants experienced a cardiovascular event during a median follow-up of 2.9 years. Age, pack-years and smoking status adjusted hazard ratios were 0.992 (95\% confidence interval (CI) 0.985-0.999) for $\mathrm{FEV}_{1} \%$ predicted, 1.000 (95\%CI 0.986-1.015) for $\mathrm{FEV}_{1} / \mathrm{FVC}, 1.014$ (95\%CI $1.005-$ 1.023 ) for perc15 per $10 \mathrm{HU}$, and 1.269 (95\%CI 1.024-1.573) for pil0 per $1 \mathrm{~mm}$. The incremental $\mathrm{C}$-index $(<0.015)$ and NRI $(<2.8 \%)$ were minimal. Coronary artery calcium volume had a hazard ratio of 1.046 (95\%CI 1.034-1.058) per $100 \mathrm{~mm}^{3}$, an increase in C-index of 0.076 and an NRI of $16.9 \%(P<0.0001)$.

Conclusions Pulmonary CT biomarkers and spirometry measurements were significantly associated with cardiovascular events, but did not contain clinically relevant independent prognostic information for cardiovascular events.

H. J. de Koning $\cdot$ C. M. van der Aalst

Department of Public Health, Erasmus Medical Center Rotterdam,

Rotterdam, The Netherlands

P. Zanen · J.-W. J. Lammers

Department of Pulmonology, University Medical Center Utrecht, Utrecht, The Netherlands

H. J. M. Groen

Department of Pulmonology, University Medical Center Groningen, Groningen, The Netherlands

E. M. van Rikxoort • B. van Ginneken

Department of Radiology, Radboud University Nijmegen Medical Centre, Nijmegen, The Netherlands

E. M. van Rikxoort • M. Schmidt • B. van Ginneken Fraunhofer MEVIS, Institute for Medical Image Computing, Bremen, Germany 
Key Points

- Pulmonary CT biomarkers and spirometry are associated with cardiovascular events

- These pulmonary measurements do not contain clinically relevant independent prognostic information

- Only coronary calcium score improved cardiovascular risk prediction above age and smoking

Keywords Cardiovascular diseases · Spirometry · Multi-detector computed tomography $\cdot$ Smoking $\cdot$ Mass screening

$\begin{array}{ll}\begin{array}{l}\text { Abbreviations } \\ \text { COPD }\end{array} & \begin{array}{l}\text { Chronic obstructive pulmonary disease } \\ \text { CT }\end{array} \\ \text { CVD } & \begin{array}{l}\text { Computed Tomography } \\ \text { FErdiovascular disease } \\ \text { FEV1 }\end{array} \\ \text { FEV1) }\end{array}$

\section{Introduction}

Lung cancer screening trials have mainly focused on the pulmonary consequences of smoking rather than cardiovascular events $[1,2]$. Smoking is strongly associated with cardiovascular events [3]. Coronary calcifications, a strong predictor for cardiovascular events $[4,5]$, can be quantified using non-contrast Computed Tomography (CT) imaging with or without ECG-gating [6, 7]. Moreover, smoking is considered the most important modifiable risk factor for developing chronic obstructive pulmonary disease (COPD) [8-10]. COPD is thought to be an independent risk factor for cardiovascular events [11]. The pathologic features of COPD are destruction of lung parenchyma (emphysema) and inflammation of the small airways (bronchiolitis). CT can clearly visualize and quantify emphysema and bronchial wall thickness and this may reflect more distal small airway diseases [12-15].

Several studies observed an association between pulmonary function and cardiovascular events, even after correcting for confounders like smoking [16-20]. Forced expiratory volume in one second $\left(\mathrm{FEV}_{1}\right)$ is a robust accurate index reflecting pulmonary physiology and is associated with a higher risk of dying from cardiovascular conditions $[17,18]$.
Moreover, the annual rate of $\mathrm{FEV}_{1}$ decline predicts cardiovascular mortality, independent of the initial predicted $\mathrm{FEV}_{1}$ and cigarette smoking $[21,22]$. On a sub-clinical level, a low $\mathrm{FEV}_{1}$ is linked to an increased mean carotid artery intimamedia thickness [23]. Despite these interesting associations it remains to be established whether these CT biomarkers or spirometry measurements do contain relevant predictive value for cardiovascular events.

The aim of our study, therefore, was to investigate the association of spirometry and pulmonary CT biomarkers with cardiovascular events and to determine the independent prognostic value of these measures for cardiovascular events.

\section{Materials and methods}

Ethics statement

This current study is an ancillary study of the Dutch and Belgian Lung Cancer Screening Trial (NELSON trial; ISRCTN63545820) approved by the Ministry of Health of The Netherlands and the institutional ethical boards of the participating centres. Written informed consent was obtained from all subjects.

\section{Participants}

A sample of Dutch and Belgian persons (50-75 years old) registered in population registries were inquired about their health and smoking behaviour [24]. Participants who had smoked $\geq 15$ cigarettes per day for 25 years or $\geq 10$ cigarettes for 30 years and were current smokers or had quit $<10$ years ago were included.

\section{Pulmonary function testing}

Spirometry was performed between 2004 and 2008 according to the American Thoracic Society and the European Respiratory Society guidelines using ZAN hardware (ZAN Messgeräte $\mathrm{GmbH}$, Oberthulba, Germany) and Viasys hardware (Viasys Health Care, Yorba Linda, CA, USA) [25]. $\mathrm{FEV}_{1}$ was expressed as percent predicted $\left(\mathrm{FEV}_{1} \%\right.$ predicted) and the $\mathrm{FEV}_{1} / \mathrm{FVC}$ ratio was determined. A bronchodilator was not administered.

\section{Computed tomography}

Participants received a volumetric non-contrast enhanced chest $\mathrm{CT}$ at full inspiration. Non-ECG-gated images were obtained on 16-slice MDCT hardware with a $16 \mathrm{~mm} \mathrm{x}$ $0.75 \mathrm{~mm}$ collimation (Sensation-16, Siemens Medical Solutions, Forchheim, Germany; Mx8000 IDT or Brilliance-16P CT, Philips Medical Systems, Best, the Netherlands). Imaging 
acquisition was performed in a cranial-caudal direction. A $120-\mathrm{kV}$ tube potential was applied in patients weighing less than $80 \mathrm{~kg}$; in patients weighing more than $80 \mathrm{~kg}$ the tube potential was increased to $140 \mathrm{kV}$. The mAs settings were dependent on the CT hardware used and adjusted according to weight.

Pulmonary emphysema and bronchial wall thickness quantification

All pulmonary CT measures were automatically quantified with CIRRUS Lung 12.03 (Diagnostic Image Analysis Group, Nijmegen, The Netherlands; Fraunhofer MEVIS, Bremen, Germany). An observer with three years experience in chest $\mathrm{CT}$ checked the lung and airway segmentations for correctness. The lungs were extracted using an automatic segmentation algorithm [26]. The density of each voxel in the segmented lung volume was determined. The density of the lungs was quantified with the 15 th percentile point (perc15). Perc15 is the threshold CT density value at which $15 \%$ of the total voxels have a lower density. The perc 15 was corrected for total lung capacity according to the following formula [27]:

Adjusted percentile density $=$ observed percentile density

$$
\times \text { (total lung volume/predicted total lung capacity). }
$$

Predicted total lung volume was calculated using the following formula [28]:

$7.99 \times($ height in $\mathrm{m})-7.08$

Airway wall thickness was calculated for each subject by taking the square root of the wall area for a theoretical airway with a 10-mm lumen perimeter (Pi10). A detailed description of the quantitative analysis of bronchial dimensions used in this study was recently published elsewhere [29].

\section{Coronary artery calcification quantification}

A coronary calcium score was automatically obtained using dedicated, in-house developed software [30]. The algorithm applies a CT threshold of $130 \mathrm{HU}$ in combination with threedimensional connected-component labelling to identify potential calcifications. Calcifications were detected based on a probabilistic coronary calcium map, followed by a pattern recognition system detecting calcifications based on size, spatial and texture characteristics. Spatial features were established using a coronary calcium atlas providing an a priori probability for spatial appearance of coronary calcifications in a chest CT. Texture features were computed using Gaussian filters at multiple scales. All coronary calcifications were visually inspected and manually corrected if deemed necessary. Coronary calcium burden was expressed in total calcium volume $\left(\mathrm{mm}^{3}\right)$.

\section{Outcome measures}

Physician-diagnosed cause of death and hospital admission diagnosis was retrieved via probabilistic medical record linkage with the National Death Registry and the National Registry of Hospital Discharge Diagnoses [31]. We included fatal and non-fatal cardiovascular events that occurred between the CT examination and January 2008. Discharge diagnoses were classified using the 9th revision of the International Classification of Diseases (ICD) and causes of death were classified using the 10th revision of the ICD. Hypertensive disease (codes 401-405), ischemic heart disease (codes 410-414), heart failure (code 428), diseases of arteries, arterioles and capillaries (codes 440-448), cerebrovascular disease (codes 430-438) or other heart diseases (code 429) were included as cardiovascular events. Cardiovascular death predominated among the hospital admissions diagnoses. In cases with several valid hospital admissions the first hospital discharge diagnosis was applied. Participants with a cardiovascular history, defined as those with a cardiovascular event between 1995 and the CT, were excluded $(n=448)$.

\section{Statistical analysis}

Normally distributed data are presented as mean \pm standard deviation (SD) and compared with the unpaired Student's t test. Non-normally distributed data were summarized as the median plus the 25th-75th percentile and compared with the Mann-Whitney U test. Normal distribution was tested evaluating Quantile-Quantile plots. Imputation based on a regression model was applied to predict missing observations. The association between the occurrence of a cardiovascular event and potential predictors was analysed using multivariable Cox proportional hazard analysis adjusting for age, smoking status and pack-years (base risk model) in order to minimize bias based on those three factors. Adjusted hazard ratios with $95 \%$ CIs were calculated. CT quantified emphysema, airway wall thickness, pulmonary function or coronary calcium volume were added separately. Coronary calcium volume was truncated at the 99th percentile (excluding 31 subjects) since biologically implausible values have a large impact on the prognostic accuracy. Bootstrap re-sampling (1,000 iterations) was applied to internally validate the predictors and adjust for over-optimism of the model. Net reclassification improvement (NRI) tables with cut points at $3 \%$ and $6 \%$ three-year risk were constructed to evaluate the added value of $\mathrm{CT}$ quantified emphysema, airway wall thickness, pulmonary function and coronary calcium volume in terms of reclassification [32]. ROCs were calculated for the different models. Kaplan-Meier survival curves for cardiovascular events were stratified by 
Table 1 Baseline patient demographics and risk factors

\begin{tabular}{lc}
\hline & $N=3080$ \\
\hline Age (year), median (25th-75th) & $59.2(55.9-63.3)$ \\
Current smoker, n (\%) & $1765(57.3)$ \\
Former smoker, n (\%) & $1315(42.7)$ \\
Pack years (year), median (25th-75th) & $38.0(28.0-49.5)$ \\
FEV ${ }_{1} \%$ predicted, $(\%)$ mean $( \pm \mathrm{SD})$ & $98.1(18.5)$ \\
FEV 1 FVC (\%), mean $( \pm \mathrm{SD})$ & $71.9(9.5)$ \\
Emphysema Percentile15 $(\mathrm{HU})$, mean $( \pm \mathrm{SD})$ & $-861.1(145.1)$ \\
Bronchial wall thickness $(\mathrm{Pi} 10)(\mathrm{mm})$, & $2.4(2.0-2.8)$ \\
$\quad$ median $(25$ th-75th) & $2.9(2.7-3.3)$ \\
Follow-up time (year), median $(25$ th-75th) & $184(6.0)$ \\
Cardiovascular event, n $(\%)$ &
\end{tabular}

quartiles of the individual predictor and a log-rank test for a linear trend was applied to test whether there was a linear trend between the quartiles. A $P$-value of 0.05 was considered statistically significant. All statistical analyses were performed using SPPS version 19 (SPSS Inc, Chicago, Illinois, USA) and $\mathrm{R}$ version 2.10.2 (R Foundation for Statistical Computing, Vienna, Austria).

\section{Results}

\section{Subject characteristics}

The analysis included 3,080 male (median age: 59.2, 25th75th percentile: $55.9-63.3)$ current $(n=1765,57.3 \%)$ and former $(n=1315,42.7 \%)$ smokers without prior cardiovascular disease (CVD) who underwent spirometry and CT lung cancer screening. Missing data were imputed for smoking status $(n=6 ; 0.2 \%)$, pack-years $(n=10 ; 0.3 \%)$, $\mathrm{FEV}_{1} \%$ predicted $(n=1 ; 0.0 \%), \mathrm{FEV}_{1} / \mathrm{FVC}(n=1 ; 0.0 \%)$, pi10 $(n=180 ; 5.8 \%)$ and perc15 $(n=158 ; 5.1 \%)$. The median follow-up period was 2.9 years (25th-75th percentile: $2.7-3.3$ ) and 184 participants experienced a cardiovascular event. Cardiovascular event-free survival was 0.956 at one year, 0.948 at two years and 0.943 at three years. Detailed patient demographics are listed in Table 1.

Pulmonary function tests

The $\mathrm{FEV}_{1} \%$ predicted $(P=0.0018)$ was significantly lower in participants who experienced an event compared to those who did not. However, $\mathrm{FEV}_{1} / \mathrm{FVC}(P=0.3918)$ was not significantly different (Table 2, Fig. 1a and b). In multivariate Cox proportional hazards models for cardiovascular events that controlled for age, smoking status and pack-years pulmonary function was a significant predictor, with a hazard ratio of 0.992 (95\% $\%$ CI 0.985-0.999) for $\mathrm{FEV}_{1} \%$ predicted and 1.000 (95\% CI 0.986-1.015) for $\mathrm{FEV}_{1} / \mathrm{FVC}$. Adding $\mathrm{FEV}_{1} \%$ predicted or $\mathrm{FEV}_{1} / \mathrm{FVC}$ to the base risk model did not yield an improved NRI at the 3-year follow-up; NRI was $1.0 \%(P=0.72)$ for $\mathrm{FEV}_{1} \%$ predicted and $-0.6 \%(P=0.56)$ for $\mathrm{FEV}_{1} / \mathrm{FVC}$ (Table 3 ). Comparison of the $\mathrm{C}$-indices confirmed this finding (Table 3 ).

\section{CT emphysema and airway wall thickness}

Measurements of emphysema and airway wall thickness were significantly worse ( $P=0.0249$ and $P=0.0074$, respectively) in participants who experienced an event (Table 2). KaplanMeier plots in Fig. 1c and d show an association between quartiles of pulmonary CT measurements and a cardiovascular event. In multivariate Cox proportional hazards models for survival outcomes that controlled for age, smoking status and pack-years pulmonary CT measurements were significant predictors, with a hazard ratio of 1.014 (95\%CI 1.005-1.023) for perc 15 per $10 \mathrm{HU}$ and 1.269 (95\%CI 1.024-1.573) for pi10 per $1 \mathrm{~mm}$. Nevertheless, adding perc 15 or pil 0 to the base risk model did not yield a significant improved risk reclassification at the 3-year follow-up: NRI $2.8 \%(P=0.41)$ and $-1.5 \%(P=$ $0.63)$, respectively. Also, the combination of perc15 and pi10

Table 2 Spirometry and CT biomarkers in lung cancer screening participants with and without incident cardiovascular events

\begin{tabular}{|c|c|c|c|c|c|}
\hline \multirow[t]{2}{*}{ Variable } & \multicolumn{2}{|c|}{ No cardiovascular event } & \multicolumn{2}{|c|}{ Incident cardiovascular event } & \multirow[t]{2}{*}{$P$ value } \\
\hline & Mean & $95 \%$-CI & Mean & $95 \%$-CI & \\
\hline $\mathrm{FEV}_{1} \%$ predicted (\%) & 98.3 & $97.7-99.0$ & 94.0 & $91.1-96.8$ & 0.0018 \\
\hline $\mathrm{FEV}_{1} / \mathrm{FVC}(\%)$ & 71.9 & $71.5-72.2$ & 71.3 & $69.7-72.8$ & 0.3918 \\
\hline Perc15 (HU) & -862.6 & $-867.9--857.3$ & -837.8 & $-856.9--818.7$ & 0.0249 \\
\hline $\operatorname{Pi} 10^{*}(\mathrm{~mm})$ & 2.4 & $2.0-2.8$ & 2.5 & $2.1-2.9$ & 0.0074 \\
\hline Coronary calcium volume $\left(\mathrm{mm}^{3}\right)^{*}$ & 122.9 & $6.2-505.6$ & 588.5 & 139.4-1432.4 & $<0.0001$ \\
\hline
\end{tabular}

*Not normal distributed median +25 th- 75 th percentile

Forced expiratory volume in one second predicted ( $\mathrm{FEV}_{1}$ \%predicted), $\mathrm{FEV}_{1}$ divided by forced vital capacity $\left(\mathrm{FEV}_{1} / \mathrm{FVC}\right)$, density of the lungs at the 15 th percentile point (perc15), bronchial wall thickness (pi10), CI Confidence interval 
a

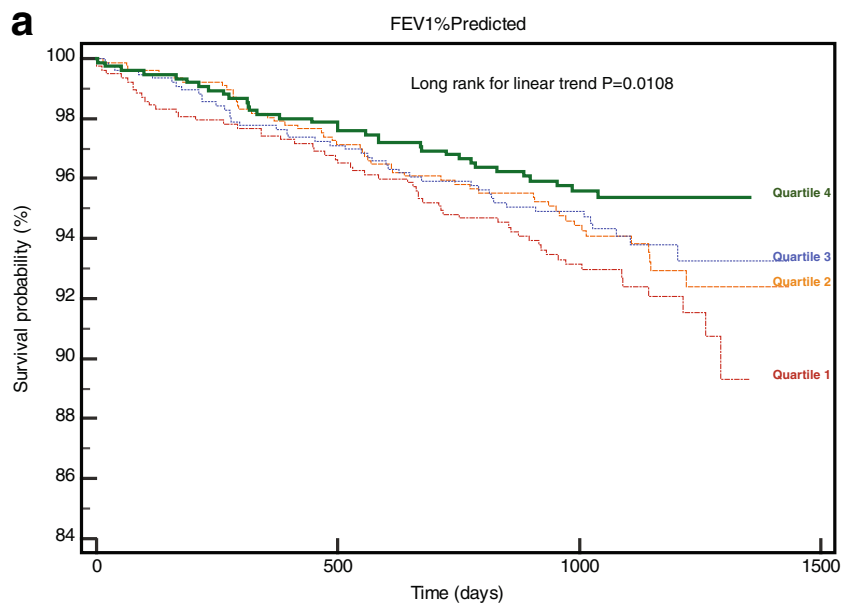

b

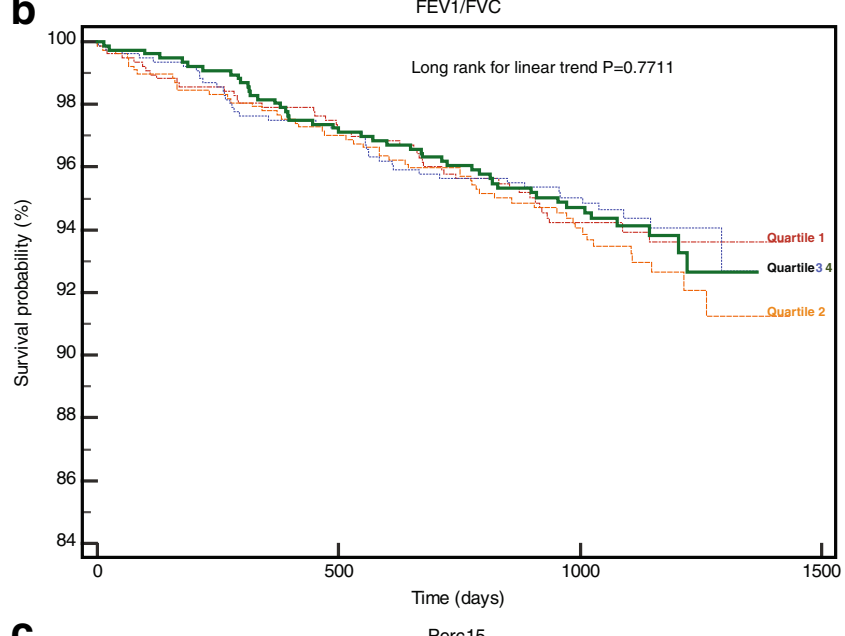

C

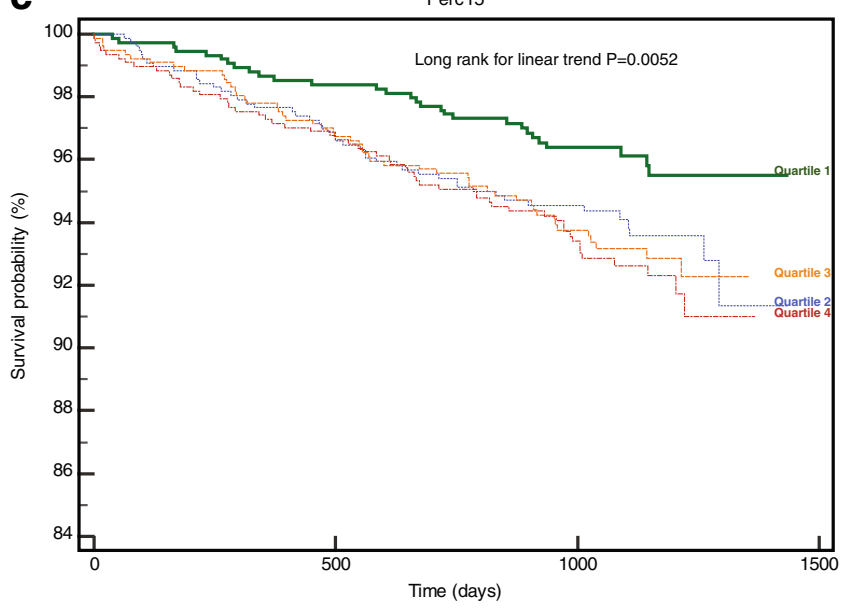

d

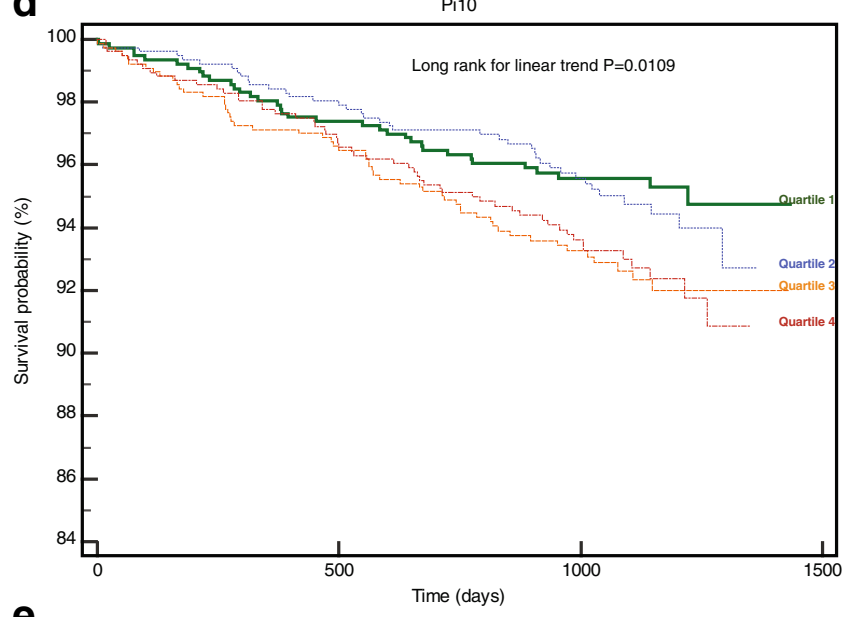

e

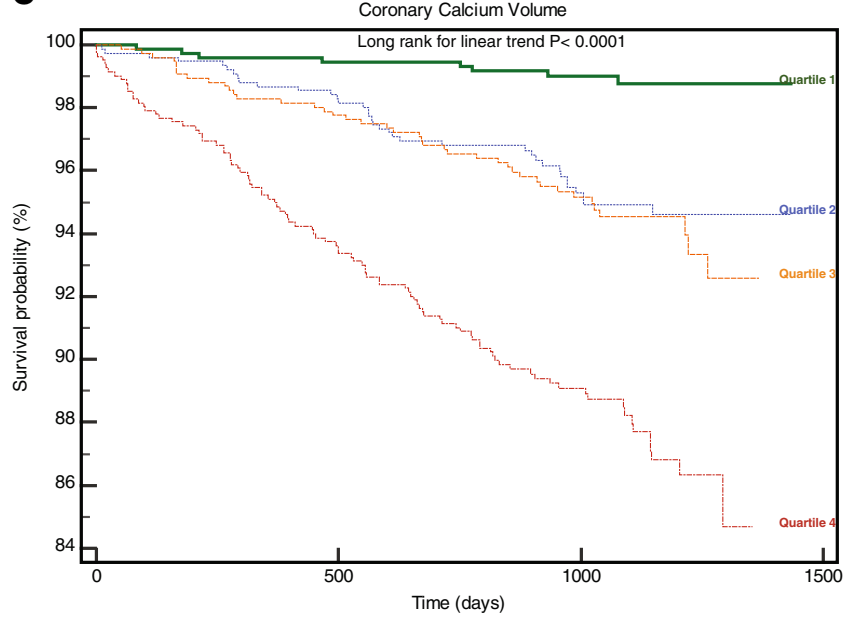

Fig. 1 Kaplan-Meier survival curves for incident cardiovascular events by quartile. (a) $\mathrm{FEV}_{1} \%$ predicted; (b) FEV $/ \mathrm{FVC}$; (c) perc15; (d) pi10; and (e) coronary calcium volume. Tests for linear trend were performed across the quartiles

did not result in a significant improvement of NRI (Table 3). The C-index curves support these observations (Table 3).

\section{CT coronary calcium score}

Coronary calcium volume was significantly $(P<0.0001)$ higher in participants who experienced an event (Table 2).
Kaplan-Meier plots in Fig. 1e show a clear association between quartiles of coronary calcium volume and a cardiovascular event. In multivariate Cox proportional hazards models that controlled for age, smoking status and pack-years the coronary calcium volume was a significant predictor for cardiovascular events, with a hazard ratio of $1.046(95 \% \mathrm{CI}$ $1.034-1.058)$ per $100 \mathrm{~mm}^{3}$. Moreover, adding the coronary 
Table 3 Prognostic performance of CT biomarkers and spirometry for incident cardiovascular disease

\begin{tabular}{lllrr}
\hline Variable & C-statistic & P-value & NRI & P-value \\
\hline Base model & 0.623 & & & \\
Base+FEV $_{1}$ \%predicted & 0.631 & 0.3772 & 1.0 & 0.7206 \\
Base+FEV $_{1} / \mathrm{FVC}$ & 0.623 & 0.5706 & -0.6 & 0.5632 \\
Base+Perc15 & 0.634 & 0.2485 & 2.8 & 0.4111 \\
Base+Pi10 & 0.626 & 0.6681 & -1.5 & 0.6316 \\
Base+Perc15+Pi10 & 0.638 & 0.2234 & 1.4 & 0.7003 \\
Base+Coronary calcium volume & 0.699 & $<0.0001$ & 16.9 & $<0.0001$ \\
\hline
\end{tabular}

Base model adjusted for age, smoking status and pack-years, forced expiratory volume in one second predicted ( $\mathrm{FEV}_{1} \%$ predicted), $\mathrm{FEV}_{1}$ divided by forced vital capacity $\left(\mathrm{FEV}_{1} / \mathrm{FVC}\right)$, density of the lungs at the 15th percentile point (perc15), bronchial wall thickness (pi10)

calcium volume to the Cox proportional hazard model containing age, pack-years and smoking status resulted in an NRI of $16.9 \%(P<0.0001)$ and an improved $\mathrm{C}$-index from 0.623 to 0.699 (Table 3).

\section{Discussion}

We found that pulmonary function as well as CT quantified emphysema and airway wall thickness in a male lung cancer screening population were associated with a modest but significant increase in cardiovascular event risk after adjustment for age, smoking status and pack-years. Nevertheless, neither pulmonary function nor CT measures of emphysema and bronchial wall thickness had sufficient independent prognostic value beyond age and smoking details. After adjustment for age, pack-years and smoking status only coronary calcium volume improved cardiovascular event prediction.

Several investigators have studied the association between pulmonary function and subsequent cardiovascular disease $[16-18,20]$. Our data are consistent with these studies as we observed clear trends across quartiles of $\mathrm{FEV}_{1} \%$ predicted, similar to Schroeder et al. [16], linking pulmonary function impairment with cardiovascular events. This association persisted after controlling for age, smoking status and packyears. Sverzellati et al. investigated the association between CT quantified emphysema and the prediction of cardiovascular events [20]. Similar to our study, they found that coronary calcium was the strongest predictor for cardiovascular events, though in contrast we did observe an association between cardiovascular events and CT quantified emphysema and FEV1\%. Also, data in a clinical population with visual scores showed that airway wall thickening is associated with future cardiovascular events [33]. Similar to CVD, COPD, emphysema and bronchiolitis are accompanied by low-grade systemic inflammation $[8,11,34]$. There is growing evidence indicating that cardiovascular disease is a major cause of death in COPD patients [35]. We confirmed that spirometry measures were associated with cardiovascular events after adjustment.

Besides confirming associations between pulmonary disease and cardiovascular events we quantified the independent value of these potential prognostic markers. Unfortunately, neither spirometry measurements nor pulmonary CT biomarkers improved cardiovascular event prediction. Coronary calcium volume, as a positive control, was the only risk factor that yielded a significant improved risk reclassification, which is consistent with previous research $[5,36]$. Prior research showed that coronary calcium is superior to standard Framingham risk factors for prediction of cardiovascular events [37]. The current study confirms the prognostic value of coronary calcifications, even from non-gated chest $\mathrm{CT}$ in a lung cancer screening setting. The conclusion is that although smokinginduced pulmonary disease is clearly associated with cardiovascular events, spirometric or CT measurements did not improve CVD prognostication in our cohort.

An important strength of our study design is the large sample of lung cancer screening participants selected from the general population. Several limitations of this study should be mentioned. First, we included only males who were current smokers or had quit less than 10 years ago. Therefore, we cannot generalize our findings to women and non-smokers. Secondly, information of a history of CVD before the year 1995 was not obtainable because linkage of patient characteristics with hospital discharge registries was impossible before that date. This probably resulted in inclusion of some patients with a cardiovascular history in our analysis.

In conclusion, our study provides further insights into the relationship between CT-quantified emphysema and airway wall thickness and cardiovascular events in asymptomatic male lung cancer screening participants. Although spirometry and pulmonary CT measurements were significantly associated with cardiovascular events, they did not yield improved cardiovascular event risk stratification and are therefore not useful prognostic CVD biomarkers.

Acknowledgments The scientific guarantor of this publication is Pim A. de Jong. The authors of this manuscript declare relationships with the following companies: HJ de Koning received money for being on the Member Advisory Board of Roche Diagnostics. This study has received funding by: The Netherlands Organisation for Health Research and Development (ZonMw); the Dutch Cancer Society; and the Koningin Wilhelmina Fonds; Stichting Centraal Fonds Reserves van Voormalig Vrijwillige Ziekenfondsverzekeringen (RVVZ); Siemens Germany (provided 4 digital workstations and LungCARE for the performance of 3D measurements); Rotterdam Oncologic Thoracic Steering Committee; and the G. Ph. Verhagen Trust, Flemish League Against Cancer, Foundation Against Cancer, and Erasmus Trust Fund. One of the authors has significant statistical expertise. Institutional Review Board approval was 
obtained. Written informed consent was obtained from all subjects (patients) in this study.

Some study subjects or cohorts have been previously reported. This study is an ancillary study of a large lung cancer screening RCT (NELSON Study; ISRCTN63545820).

Methodology: prospective, prognostic study (original study was a randomised lung cancer screening trial), multi-center study.

\section{References}

1. Mets OM, de Jong PA, Prokop M (2012) Computed tomographic screening for lung cancer: an opportunity to evaluate other diseases. JAMA 308:1433-1434

2. National Lung Screening Trial Research T, Aberle DR, Adams AM (2011) Reduced lung-cancer mortality with low-dose computed tomographic screening. N Engl J Med 365:395-409

3. Burns DM (2003) Epidemiology of smoking-induced cardiovascular disease. Prog Cardiovasc Dis 46:11-29

4. Becker CR (2005) Estimation of cardiac event risk by MDCT. Eur Radiol 15:B17-B22

5. Vliegenthart R, Oudkerk M, Hofman A et al (2005) Coronary calcification improves cardiovascular risk prediction in the elderly. Circulation 112:572-577

6. Mets OM, Vliegenthart R, Gondrie MJ et al (2013) Lung Cancer Screening CT-Based Prediction of Cardiovascular Events. JACC Cardiovasc Imaging 6:899-907

7. Xie X, Zhao Y, de Bock GH et al (2013) Validation and Prognosis of Coronary Artery Calcium Scoring inNon-Triggered Thoracic Computed Tomography: Systematic Review and Meta-Analysis. Circ Cardiovasc Imaging 6:514-521

8. Wouters EF (2005) Local and systemic inflammation in chronic obstructive pulmonary disease. Proc Am Thorac Soc 2:26-33

9. Stone IS, Barnes NC, Petersen SE (2012) Chronic obstructive pulmonary disease: a modifiable risk factor for cardiovascular disease? Heart 98:1055-1062

10. Bellomi M, Rampinelli C, Veronesi G et al (2010) Evolution of emphysema in relation to smoking. Eur Radiol 20:286-292

11. Sin DD, Man SF (2003) Why are patients with chronic obstructive pulmonary disease at increased risk of cardiovascular diseases? The potential role of systemic inflammation in chronic obstructive pulmonary disease. Circulation 107:1514-1519

12. Mets OM, Murphy K, Zanen P et al (2012) The relationship between lung function impairment and quantitative computed tomography in chronic obstructive pulmonary disease. Eur Radiol 22:120-128

13. Mohamed Hoesein FA, de Hoop B, Zanen P et al (2011) CTquantified emphysema in male heavy smokers: association with lung function decline. Thorax 66:782-787

14. Nakano Y, Wong JC, de Jong PA et al (2005) The prediction of small airway dimensions using computed tomography. Am J Respir Crit Care Med 171:142-146

15. Camiciottoli G, Cavigli E, Grassi L et al (2009) Prevalence and correlates of pulmonary emphysema in smokers and former smokers. A densitometric study of participants in the ITALUNG trial. Eur Radiol 19:58-66

16. Schroeder EB, Welch VL, Couper D et al (2003) Lung function and incident coronary heart disease: the Atherosclerosis Risk in Communities Study. Am J Epidemiol 158:1171-1181

17. Stavem K, Aaser E, Sandvik L et al (2005) Lung function, smoking and mortality in a 26-year follow-up of healthy middle-aged males. Eur Respir J 25:618-625

18. Hole DJ, Watt GC, Davey-Smith G, Hart CL, Gillis CR, Hawthorne VM (1996) Impaired lung function and mortality risk in men and women: findings from the Renfrew and Paisley prospective population study. BMJ 313:711-715, discussion 715-716

19. Johnston AK, Mannino DM, Hagan GW, Davis KJ, Kiri VA (2008) Relationship between lung function impairment and incidence or recurrence of cardiovascular events in a middle-aged cohort. Thorax 63:599-605

20. Sverzellati N, Cademartiri F, Bravi F et al (2012) Relationship and prognostic value of modified coronary artery calcium score, FEV1, and emphysema in lung cancer screening population: the MILD trial. Radiology 262:460-467

21. Tockman MS, Pearson JD, Fleg JL et al (1995) Rapid decline in FEV1. A new risk factor for coronary heart disease mortality. Am J Respir Crit Care Med 151:390-398

22. Ryan G, Knuiman MW, Divitini ML, James A, Musk AW, Bartholomew HC (1999) Decline in lung function and mortality: the Busselton Health Study. J Epidemiol Community Health 53:230-234

23. Iwamoto H, Yokoyama A, Kitahara Y et al (2009) Airflow limitation in smokers is associated with subclinical atherosclerosis. Am J Respir Crit Care Med 179:35-40

24. van den Bergh KA, Essink-Bot ML, Borsboom GJ et al (2010) Shortterm health-related quality of life consequences in a lung cancer $\mathrm{CT}$ screening trial (NELSON). Br J Cancer 102:27-34

25. Miller MR, Crapo R, Hankinson J et al (2005) General considerations for lung function testing. Eur Respir J 26:153-161

26. van Rikxoort EM, de Hoop B, Viergever MA, Prokop M, van Ginneken B (2009) Automatic lung segmentation from thoracic computed tomography scans using a hybrid approach with error detection. Med Phys 36:2934-2947

27. Dirksen A (2008) Monitoring the progress of emphysema by repeat computed tomography scans with focus on noise reduction. Proc Am Thorac Soc 5:925-928

28. Quanjer PH, Tammeling GJ, Cotes JE, Pedersen OF, Peslin R, Yernault JC (1993) Lung volumes and forced ventilatory flows. Report Working Party Standardization of Lung Function Tests, European Community for Steel and Coal. Official Statement of the European Respiratory Society. Eur Respir J Suppl 16:5-40

29. Mets OM, Schmidt M, Buckens CF et al (2013) Diagnosis of chronic obstructive pulmonary disease in lung cancer screening Computed Tomography scans: independent contribution of emphysema, air trapping and bronchial wall thickening. Respir Res 14:59

30. Isgum I, Prokop M, Niemeijer M, Viergever MA, van Ginneken B (2012) Automatic coronary calcium scoring in low-dose chest computed tomography. IEEE Trans Med Imaging 31:2322-2334

31. Tromp M, Ravelli AC, Bonsel GJ, Hasman A, Reitsma JB (2011) Results from simulated data sets: probabilistic record linkage outperforms deterministic record linkage. J Clin Epidemiol 64:565-572

32. Pencina MJ, D'Agostino RB Sr, D'Agostino RB Jr, Vasan RS (2008) Evaluating the added predictive ability of a new marker: from area under the ROC curve to reclassification and beyond. Stat Med 27: 157-172, discussion 207-112

33. de Jong PA, Gondrie MJ, Buckens CF et al (2011) Prediction of cardiovascular events by using non-vascular findings on routine chest CT. PLoS One 6:e26036

34. Vanfleteren LE, Spruit MA, Groenen M et al (2013) Clusters of comorbidities based on validated objective measurements and systemic inflammation in patients with chronic obstructive pulmonary disease. Am J Respir Crit Care Med 187:728-735

35. Sin DD, Anthonisen NR, Soriano JB, Agusti AG (2006) Mortality in COPD: Role of comorbidities. Eur Respir J 28:1245-1257

36. Jacobs PC, Gondrie MJ, van der Graaf Y et al (2012) Coronary artery calcium can predict all-cause mortality and cardiovascular events on low-dose CT screening for lung cancer. AJR Am J Roentgenol 198: 505-511

37. Polonsky TS, McClelland RL, Jorgensen NW et al (2010) Coronary artery calcium score and risk classification for coronary heart disease prediction. JAMA 303:1610-1616 